\title{
Actualización: Toma de decisiones para la prevención y el tratamiento de la enfermedad cardiovascular
}

\author{
Decision-making for prevention and treatment of cardiovascular disease
}

Sergio Terrasa*

\begin{abstract}
Resumen
En dos artículos publicados durante 2003 en EVIDENCIA hicimos hincapié en lo que en aquellos años comenzaba a perfilarse como el nuevo paradigma para la toma de decisiones en prevención cardiovascular (CV) primaria y secundaria; ofrecimos a los lectores una adaptación en castellano de tres herramientas derivadas de sendas cohortes para estimar riesgo futuro de que un paciente individual desarrolle enfermedad CV; y resumimos las metas de tratamiento recomendadas por los principales consensos para las diferentes poblaciones según su riesgo CV.

En este artículo resumimos y discutimos las recomendaciones de la nueva guía de la Organización Mundial de la Salud publicada en 2007, que teniendo en cuenta la finitud de los recursos en salud, propone a similares niveles de riesgo CV, metas menos estrictas y por lo tanto intervenciones menos intensivas que las recomendadas por los principales comités de expertos para el manejo de la hipertensión arterial, la dislipidemia y la diabetes.
\end{abstract}

\begin{abstract}
In two papers published in EVIDENCIA in 2003 we highlighted what in that time was surging as a decision-making paradigm for primary and secondary cardiovascular (CV) disease prevention; and we offered our readers a Spanish language adaptation of three tools derived from cohort studies that estimated the future risk of having CV disease; and we also summarized treatment goals recommended by the main guidelines for populations with different CV risk. In this article we synthesize and discuss the new recommendations from the W.H.O guideline published in 2007, that takes into consideration the scarcity of resources, proposes less intensive goals for similar CV risks as well as less intensive interventions than those recommended by the main high blood pressure, high cholesterol or diabetes guidelines.
\end{abstract}

Palabras clave: riesgo cardiovascular, enfermedad cardiovascular, prevención, terapéutica. Key words: cardiovascular risk, cardiovascular disease, prevention, treatment.

Terrasa S. Toma de decisiones para la prevención y el tratamiento de la enfermedad cardiovascular. Evid Act Pract Ambul. 11(3) 87-91. May-Jun 2008.

\section{El contexto epidemiológico}

La enfermedad cardiovascular (CV) es responsable del $30 \%$ de la mortalidad a nivel mundial, ocurriendo el $46 \%$ de estas muertes en personas económicamente activas y dando cuenta las afecciones cardiovasculares del $79 \%$ de la carga de enfermedad de las personas menores de 70 años. Si bien las manifestaciones clínicas de la enfermedad CV comienzan en la adultez y se incrementan en forma directa con la edad, su fisiopatogenia comienza durante la niñez y la adolescencia, con fuerte asociación ampliamente documentada a varios factores de riesgo modificables: básicamente tabaquismo, hipertensión arterial, diabetes y dislipemia y en forma algo más indirecta sedentarismo, obesidad, dieta pobre en vegetales.

Respecto de la realidad epidemiológica de nuestra región, vale la pena describir los principales resultados del estudio conducido por Schargrodsky y col. ${ }^{2}$ que evaluó mediante una técnica de muestreo polietápico estratificado*, la prevalencia de factores de riesgo CV en 11550 personas residentes en grandes ciudades de Latinoamérica (ver tabla 1).

Ver glosario*

Tabla 1: prevalencia de factores mayores de riesgo cardiovascular por ciudad de acuerdo a los resultados del estudio

\begin{tabular}{|c|c|c|c|c|c|c|c|c|}
\hline & & $\begin{array}{l}\text { Barquisimeto, } \\
\text { Venezuela }\end{array}$ & $\begin{array}{c}\text { Bogotá, } \\
\text { Colombia }\end{array}$ & $\begin{array}{l}\text { Buenos Aires, } \\
\text { Argentina }\end{array}$ & $\begin{array}{l}\text { Lima, } \\
\text { Perú }\end{array}$ & $\begin{array}{l}\text { México DF, } \\
\text { México }\end{array}$ & $\begin{array}{l}\text { Quito, } \\
\text { Ecuador }\end{array}$ & $\begin{array}{l}\text { Santiago, } \\
\text { Chile }\end{array}$ \\
\hline \multicolumn{2}{|l|}{ Hi pertensión ${ }^{a}$} & $24,7 \%$ & $13,4 \%$ & $29 \%$ & $12,6 \%$ & $11,7 \%$ & $8,6 \%$ & $23,8 \%$ \\
\hline \multicolumn{2}{|l|}{ Hi percolesterolemiab } & $5,7 \%$ & $12 \%$ & $18,7 \%$ & $11,6 \%$ & $16,4 \%$ & $20,2 \%$ & $15,3 \%$ \\
\hline \multicolumn{2}{|l|}{ Tabaquismo $^{\circ}$} & $21,8 \%$ & $22,2 \%$ & $38,6 \%$ & $26,6 \%$ & $27,3 \%$ & $29,9 \%$ & $45,4 \%$ \\
\hline \multicolumn{2}{|l|}{ Diabetes $^{\mathrm{d}}$} & $6 \%$ & $8,1 \%$ & $6,2 \%$ & $4,4 \%$ & $8,9 \%$ & $5,9 \%$ & $7,2 \%$ \\
\hline \multicolumn{2}{|l|}{$\overline{\text { Obesidad }^{\circ}}$} & $25,1 \%$ & $18 \%$ & $19,7 \%$ & $22,3 \%$ & $31 \%$ & $16,3 \%$ & $26,6 \%$ \\
\hline \multicolumn{2}{|l|}{ Sndrome metabólico ${ }^{\prime}$} & $25,8 \%$ & $20,4 \%$ & $16,7 \%$ & $17,9 \%$ & $27,2 \%$ & $13,7 \%$ & $21 \%$ \\
\hline \multirow{3}{*}{$\begin{array}{l}\text { Riesgo cardio- } \\
\text { vascular estimado } \\
\text { a diez años }\end{array}$} & Bajo (menor a $10 \%$ ) & $88,6 \%$ & $84,8 \%$ & $82,9 \%$ & $86,7 \%$ & $85,5 \%$ & $83,2 \%$ & $82 \%$ \\
\hline & Intermedio (10 a $20 \%)$ & $2,9 \%$ & $4,5 \%$ & $5 \%$ & $4,1 \%$ & $3,8 \%$ & $5,5 \%$ & $6 \%$ \\
\hline & Alto (mayor a $20 \%$ ) & $8,5 \%$ & $10,7 \%$ & $12,1 \%$ & $9,2 \%$ & $10,7 \%$ & $11,2 \%$ & $12 \%$ \\
\hline
\end{tabular}

a Tensión arterial mayor a 140/90 mmHg o uso de drogas antihipertensivas. ${ }^{\circ}$ Colesterol total sérico mayor a $240 \mathrm{mg} / \mathrm{dL}$. ${ }^{\circ}$ Consumo actual de tabaco (cigarrillos, cigarros o pipa) diaria u ocasionalmente. 'Índice de masa corporal mayor a 30kg/m2. 'Tres o más criterios de los siguientes: obesidad abdominal (perímetro abdominal mayor a $102 \mathrm{~cm}$ en varones y $88 \mathrm{~cm}$ en mujeres) trigliceridemia mayor a 150mg/dL, colesterol HDL menor a $40 \mathrm{mg} / \mathrm{dL}$ en varones y a $50 \mathrm{mg} / \mathrm{dL}$ en mujeres, tensión arterial mayor a $130 / 85 \mathrm{mmHg}$ y glucemia en ayunas mayor a $110 \mathrm{mg} / \mathrm{dL}$. ${ }^{9}$ De acuerdo al puntaje de Framingham y definido como riesgo de infarto agudo de miocardio, angina de pecho o muerte coronaria.

Fuente: Schargrodsky H. CARMELA: Assessment of Cardiovascular Risk in Seven Latin American Cities. American Journal of Medicine (2008) 121, $58-65$.

* Servicio de Medicina Familiar y Comunitaria del Hospital Italiano de Buenos Aires sergio.terrasa@ hospitalitaliano.org.ar 


\section{Los posibles abordajes para la estimación del riesgo}

En los últimos años ha ganado terreno el abordaje de la prevención $\mathrm{CV}$ en el contexto de la valoración del riesgo global del individuo de desarrollar estas enfermedades, de acuerdo a la presencia y a la magnitud de sus factores de riesgo. El uso de puntajes para estratificar el riesgo CV fue un avance filosófico y operativo respecto de la estrategia previa de considerar a los factores de riesgo en forma aislada y dicotómica, ya que en realidad, estos tienden a comportarse en forma lineal e interaccionando entre si.

En dos artículos ${ }^{3,4}$ publicados durante $2003^{\S}$ en EVIDENCIA hicimos hincapié en lo que en aquellos años comenzaba a perfilarse como el nuevo paradigma para la toma de decisiones en prevención CV primaria y secundaria y ofrecimos a los lectores una adaptación en castellano de tres herramientas derivadas de sendas cohortes para estimar riesgo futuro de que un paciente individual desarrolle enfermedad CV: los puntajes derivados de la cohorte de Framingham (EEUU) de PROCAM (Alemania) y del proyecto $\mathrm{SCORE}^{7}$ para ser aplicado en poblaciones de Europa Meridional (Portugal, España, Francia el Italia). Estos puntajes requieren información clínica como la edad, el sexo, el consumo de tabaco, la condición de diabético, la tensión arterial, los niveles de colesterol total y colesterol HDL; y ayudan a discriminar el riesgo CV en los individuos en quienes éste no es evidentemente bajo (ej. personas jóvenes sin factores de riesgo CV; o evidentemente alto (ej. sujetos con enfermedad CV ya establecida, diabetes con mal control, múltiples factores de riesgo).

Vale destacar que la mayoría de las reglas de predicción derivadas de la cohorte de Framingham han sido validadas en EEUU, así como en poblaciones de origen europeo y chino, y tienden a sobreestimar el riesgo en poblaciones de bajo riesgo y a subestimarlo en las de muy alto riesgo, por eso es que volvemos a insistir que su aplicación puede ser de utilidad en los sujetos de riesgo intermedio.

Tabla 2: evaluación del riesgo de enfermedad cardiovascular fatal y no fatal a cinco años en mujeres sin determinación del colesterol sérico.

\begin{tabular}{|c|c|c|c|c|c|c|c|c|c|c|c|c|c|c|c|c|c|c|}
\hline \multirow{3}{*}{\begin{tabular}{|c|}
$\begin{array}{c}\text { TA en } \\
\text { mmHg }\end{array}$ \\
$171-180$ \\
\end{tabular}} & \multirow{2}{*}{\multicolumn{8}{|c|}{ No diabetes }} & Edad & \multicolumn{8}{|c|}{ Diabetes } & \multirow{3}{*}{$\begin{array}{c}\begin{array}{c}\text { TA en } \\
\text { mmHg }\end{array} \\
171-180 \\
\end{array}$} \\
\hline & \multicolumn{2}{|c|}{ No fumadoras } & & & \multicolumn{4}{|c|}{ Fumadoras } & \multirow{8}{*}{65 a 74} & \multicolumn{4}{|c|}{ No fumadoras } & \multicolumn{4}{|c|}{ Fumadoras } & \\
\hline & $20-30$ & \begin{tabular}{|l|}
$20-30$ \\
\end{tabular} & $>30$ & $>30$ & $>30$ & $>30$ & $>30$ & $>30$ & & $>30$ & $>30$ & $>30$ & $>30$ & $>30$ & $>30$ & $>30$ & $>30$ & \\
\hline 161-170 & $20-30$ & $20-30$ & $>30$ & $>30$ & $>30$ & $>30$ & $>30$ & $>30$ & & $>30$ & $>30$ & $>30$ & $>30$ & $>30$ & $>30$ & $>30$ & $>30$ & $161-170$ \\
\hline $151-160$ & $20-30$ & $20-30$ & $20-30$ & $>30$ & $>30$ & $>30$ & $>30$ & $>30$ & & $>30$ & $>30$ & $>30$ & $>30$ & $>30$ & $>30$ & $>30$ & $>30$ & $151-160$ \\
\hline 141-150 & $20-30$ & $20-30$ & $20-30$ & $>30$ & $>30$ & $>30$ & $>30$ & $>30$ & & $>30$ & $>30$ & $>30$ & $>30$ & $>30$ & $>30$ & $>30$ & $>30$ & $141-150$ \\
\hline $131-120$ & $10-20$ & $20-30$ & $20-30$ & $20-30$ & $>30$ & $>30$ & $>30$ & $>30$ & & $>30$ & $>30$ & $>30$ & $>30$ & $>30$ & $>30$ & $>30$ & $>30$ & $131-120$ \\
\hline $121-130$ & $10-20$ & $10-20$ & $20-30$ & $20-30$ & $20-30$ & $>30$ & $>30$ & $>30$ & & $>30$ & $>30$ & $>30$ & $>30$ & $>30$ & $>30$ & $>30$ & $>30$ & $121-130$ \\
\hline $111-120$ & $10-20$ & $10-20$ & $10-20$ & $20-30$ & $20-30$ & $20-30$ & $>30$ & $>30$ & & $20-30$ & $>30$ & $>30$ & $>30$ & $>30$ & $>30$ & $>30$ & $>30$ & $111-120$ \\
\hline 171-180 & $10-20$ & $10-20$ & $20-30$ & $20-30$ & $20-30$ & $20-30$ & $>30$ & $>30$ & & $20-30$ & $20-30$ & $>30$ & $>30$ & $>30$ & $>30$ & $>30$ & $>30$ & $171-180$ \\
\hline 161-170 & $10-20$ & $10-20$ & $10-20$ & $20-30$ & $20-30$ & $20-30$ & $>30$ & $>30$ & & $20-30$ & $20-30$ & $>30$ & $>30$ & $>30$ & $>30$ & $>30$ & $>30$ & $161-170$ \\
\hline $151-160$ & $10-20$ & $10-20$ & $10-20$ & $20-30$ & $20-30$ & $20-30$ & $20-30$ & $>30$ & & $20-30$ & $20-30$ & $20-30$ & $>30$ & $>30$ & $>30$ & $>30$ & $>30$ & $151-160$ \\
\hline $141-150$ & $10-20$ & $10-20$ & $10-20$ & $10-20$ & $20-30$ & $20-30$ & $20-30$ & $>30$ & 55 a 64 & $20-30$ & $20-30$ & $20-30$ & $>30$ & $>30$ & $>30$ & $>30$ & $>30$ & $141-150$ \\
\hline $131-120$ & $10-20$ & $10-20$ & $10-20$ & $10-20$ & $10-20$ & $20-30$ & $20-30$ & \begin{tabular}{|l|}
$20-30$ \\
\end{tabular} & & $20-30$ & $20-30$ & $20-30$ & $20-30$ & $>30$ & $>30$ & $>30$ & $>30$ & $131-120$ \\
\hline $121-130$ & $10-20$ & $10-20$ & $10-20$ & $10-20$ & $10-20$ & $10-20$ & $20-30$ & 20-30 & & $10-20$ & $20-30$ & $20-30$ & $20-30$ & $>30$ & $>30$ & $>30$ & $>30$ & $121-130$ \\
\hline $111-120$ & $5-10$ & $5-10$ & $10-20$ & $10-20$ & $10-20$ & $10-20$ & $10-20$ & 20-30 & & $10-20$ & $10-20$ & $20-30$ & $20-30$ & $20-30$ & $>30$ & $>30$ & $>30$ & $111-120$ \\
\hline $171-180$ & $5-10$ & $5-10$ & 10-20 & 10-20 & $10-20$ & $10-20$ & $10-20$ & \begin{tabular}{|l|}
$\mid 20-30$ \\
\end{tabular} & & $10-20$ & 10-20 & 20-30 & $20-30$ & $20-30$ & $20-30$ & $>30$ & $>30$ & $171-180$ \\
\hline 161-170 & $5-10$ & $5-10$ & 10-20 & $10-20$ & $10-20$ & $10-20$ & $10-20$ & \begin{tabular}{|l|}
$20-30$ \\
\end{tabular} & & \begin{tabular}{|l|}
$10-20$ \\
\end{tabular} & $10-20$ & $10-20$ & $20-30$ & $20-30$ & $20-30$ & $>30$ & $>30$ & $161-170$ \\
\hline $151-160$ & $5-10$ & $5-10$ & $5-10$ & $10-20$ & $10-20$ & $10-20$ & $10-20$ & \begin{tabular}{|l|}
$10-20$ \\
\end{tabular} & & $10-20$ & $10-20$ & $10-20$ & $20-30$ & $20-30$ & $20-30$ & $20-30$ & $>30$ & $151-160$ \\
\hline $141-150$ & $5-10$ & $5-10$ & $5-10$ & $10-20$ & $10-20$ & $10-20$ & $10-20$ & \begin{tabular}{|l|}
$10-20$ \\
\end{tabular} & 45 a 54 & $10-20$ & $10-20$ & $10-20$ & $10-20$ & $20-30$ & $20-30$ & $20-30$ & $>30$ & $141-150$ \\
\hline $131-120$ & $5-10$ & $5-10$ & $5-10$ & $5-10$ & $10-20$ & $10-20$ & $10-20$ & 10-20 & & $10-20$ & $10-20$ & $10-20$ & $10-20$ & $10-20$ & $20-30$ & $20-30$ & $20-30$ & $131-120$ \\
\hline $121-130$ & $5-10$ & $5-10$ & $5-10$ & $5-10$ & $5-10$ & $5-10$ & $10-20$ & 10-20 & & $5-10$ & $10-20$ & $10-20$ & $10-20$ & $10-20$ & $10-20$ & $20-30$ & $20-30$ & $121-130$ \\
\hline $111-120$ & $<5$ & $5-10$ & $5-10$ & $5-10$ & $5-10$ & $5-10$ & $10-20$ & 10-20 & & $5-10$ & $5-10$ & $10-20$ & $10-20$ & $10-20$ & $10-20$ & $10-20$ & $20-30$ & $111-120$ \\
\hline $171-180$ & $<5$ & $<5$ & $<5$ & $5-10$ & $5-10$ & $5-10$ & $5-10$ & \begin{tabular}{|l|}
$10-20$ \\
\end{tabular} & & \begin{tabular}{|l|}
$5-10$ \\
\end{tabular} & $5-10$ & $5-10$ & $10-20$ & $10-20$ & $10-20$ & $10-20$ & \begin{tabular}{|l|}
$10-20$ \\
\end{tabular} & $171-180$ \\
\hline $161-170$ & $<5$ & $<5$ & $<5$ & $5-10$ & $5-10$ & $5-10$ & $5-10$ & $5-10$ & & \begin{tabular}{|l|}
$5-10$ \\
\end{tabular} & $5-10$ & $5-10$ & $10-20$ & $10-20$ & $10-20$ & $10-20$ & \begin{tabular}{|l|}
$10-20$ \\
\end{tabular} & $161-170$ \\
\hline $151-160$ & $<5$ & $<5$ & $<5$ & $<5$ & $5-10$ & $5-10$ & $5-10$ & $5-10$ & & $5-10$ & $5-10$ & $5-10$ & $5-10$ & $10-20$ & $10-20$ & $10-20$ & \begin{tabular}{|l|}
$10-20$ \\
\end{tabular} & $151-160$ \\
\hline $141-150$ & $<5$ & $<5$ & $<5$ & $<5$ & $<5$ & $5-10$ & $5-10$ & $5-10$ & 35 a & $5-10$ & $5-10$ & $5-10$ & $5-10$ & $5-10$ & $10-20$ & $10-20$ & |10-20 & $141-150$ \\
\hline $131-120$ & $<5$ & $<5$ & $<5$ & $<5$ & $<5$ & $<5$ & $5-10$ & $5-10$ & & $<5$ & $5-10$ & $5-10$ & $5-10$ & $5-10$ & $5-10$ & $10-20$ & $10-20$ & $131-120$ \\
\hline $121-130$ & $<5$ & $<5$ & $<5$ & $<5$ & $<5$ & $<5$ & 5-10 & $5-10$ & & $<5$ & $<5$ & $5-10$ & $5-10$ & $5-10$ & $5-10$ & $5-10$ & $10-20$ & $121-130$ \\
\hline $111-120$ & $<5$ & $<5$ & $<5$ & $<5$ & $<5$ & $<5$ & $<5$ & $5-10$ & & $<5$ & $<5$ & $<5$ & $5-10$ & $5-10$ & $5-10$ & $5-10$ & $10-20$ & $111-120$ \\
\hline & $\begin{array}{c}15 \mathrm{a} \\
20\end{array}$ & $\begin{array}{c}20 \mathrm{a} \\
25\end{array}$ & $\begin{array}{c}25 \mathrm{a} \\
30\end{array}$ & $\begin{array}{c}30 \mathrm{a} \\
40\end{array}$ & $\begin{array}{c}15 a \\
20\end{array}$ & $\begin{array}{c}20 \mathrm{a} \\
25\end{array}$ & $\begin{array}{c}25 \mathrm{a} \\
30\end{array}$ & \begin{tabular}{|c|}
$30 \mathrm{a}$ \\
40
\end{tabular} & & $\begin{array}{c}15 \mathrm{a} \\
20\end{array}$ & $\begin{array}{c}20 a \\
25\end{array}$ & $\begin{array}{c}25 \mathrm{a} \\
30\end{array}$ & $\begin{array}{c}30 \mathrm{a} \\
40\end{array}$ & $\begin{array}{c}15 \mathrm{a} \\
20\end{array}$ & $\begin{array}{c}20 \mathrm{a} \\
25\end{array}$ & $\begin{array}{c}25 \mathrm{a} \\
30\end{array}$ & $\begin{array}{c}30 \mathrm{a} \\
40\end{array}$ & \\
\hline & & & & & & & & & & & & & & & & $g / m^{2}$ & & \\
\hline
\end{tabular}

$\S$ Si el lector está poco familiarizado con este tipo de abordaje, recomendamos que antes de leer este artículo lea estos documentos. 
Más allá de las recomendaciones generales como el consejo anti-tabaco, el estímulo de una práctica regular de actividad física y la adopción de una dieta rica en vegetales; el objetivo de "afinar" esta discriminación en el riesgo CV es contribuir a que la toma decisiones sobre la aplicación y la intensidad de las intervenciones individuales sean lo más costo-efectivas posibles.

En este sentido nos referimos a las intervenciones individuales destinadas a que una persona descienda su tensión arterial y sus lípidos -lo que puede implicar la prescripción de tratamientos farmacológicos durante tiempos prolongados-; así como a la indicación de aspirina y/o de bloqueantes de la enzima convertidora de angiotensina.

Durante estos últimos años, varios consensos $8,9,10,11,12,13,14,15$ avalaron con mayor o menor intensidad este tipo de estrategia de toma de decisiones para la prevención y el tratamiento de la enfermedad CV, varios de los cuales fueron resumidos, comentados y/o discutidos en EVIDENCIA.
En este número de EVIDENCIA acercamos al lector una nueva herramienta para la estimación individual del riesgo CV global. Su principal diferencia y ventaja radica en que no requiere la medición de los niveles de colesterol sanguíneo, pudiendo facilitar la toma de decisiones en contextos de baja accesibilidad a esta tecnología.

Ver tablas 2 y 3 . Su poder discriminatorio es aceptable y comparable a los puntajes que publicamos previamente.

Lamentablemente, todavía no contamos con herramientas construidas sobre datos epidemiológicos locales, básicamente porque todavía no existen cohortes de nuestro medio que permitan documentar el verdadero pronóstico $\mathrm{CV}$ en las distintas poblaciones de acuerdo a sus factores de riesgo.

Mencionamos, sin embargo que la Organización Mundial de la Salud (OMS) ha anunciado el desarrollo de un puntaje clínico con "correcciones" para "las Américas" con el objetivo de que ayude al equipo de salud en la toma de decisiones para la prevención CV individual y que todavía se encuentra "en prensa"16.

Tabla 3: evaluación del riesgo de enfermedad cardiovascular fatal y no fatal a cinco años en varones sin determinación del colesterol sérico.

\begin{tabular}{|c|c|c|c|c|c|c|c|c|c|c|c|c|c|c|c|c|c|c|}
\hline \multirow{3}{*}{\begin{tabular}{|c|}
$\begin{array}{c}\text { TA en } \\
\text { mmHg }\end{array}$ \\
$171-180$
\end{tabular}} & \multicolumn{8}{|c|}{ No diabetes } & Edad & \multicolumn{8}{|c|}{ Diabetes } & \multirow{3}{*}{\begin{tabular}{|c|}
$\begin{array}{c}\text { TA en } \\
\text { mmHg }\end{array}$ \\
$171-180$
\end{tabular}} \\
\hline & \multicolumn{4}{|c|}{ No fumadoras } & \multicolumn{4}{|c|}{ Fumadoras } & \multirow{8}{*}{65 a 74} & \multicolumn{4}{|c|}{ No fumadoras } & \multicolumn{4}{|c|}{ Fumadoras } & \\
\hline & $>30$ & $>30$ & $>30$ & $>30$ & $>30$ & $>30$ & $>30$ & $>30$ & & $>30$ & $>30$ & $>30$ & $\mid>30$ & $>30$ & $>30$ & $>30$ & $>30$ & \\
\hline 161-170 & $>30$ & $>30$ & $>30$ & $>30$ & $>30$ & $>30$ & $>30$ & $>30$ & & $>30$ & $>30$ & $>30$ & $>30$ & $>30$ & $>30$ & $>30$ & $>30$ & 161-170 \\
\hline $151-160$ & $20-30$ & $>30$ & $>30$ & $>30$ & $>30$ & $>30$ & $>30$ & $>30$ & & $>30$ & $>30$ & $>30$ & $>30$ & $>30$ & $>30$ & $>30$ & $>30$ & $151-160$ \\
\hline 141-150 & $20-30$ & $20-30$ & $>30$ & $>30$ & $>30$ & $>30$ & $>30$ & $>30$ & & $>30$ & $>30$ & $>30$ & $>30$ & $>30$ & $>30$ & $>30$ & $>30$ & 141-150 \\
\hline $131-120$ & $20-30$ & $20-30$ & $20-30$ & $>30$ & $>30$ & $>30$ & $>30$ & $>30$ & & $>30$ & $>30$ & $>30$ & $>30$ & $>30$ & $>30$ & $>30$ & $>30$ & $131-120$ \\
\hline $121-130$ & $20-30$ & $20-30$ & $20-30$ & $20-30$ & $>30$ & $>30$ & $>30$ & $>30$ & & $>30$ & $>30$ & $>30$ & $>30$ & $>30$ & $>30$ & $>30$ & $>30$ & $121-130$ \\
\hline $111-120$ & $10-20$ & $20-30$ & $20-30$ & $20-30$ & $>30$ & $>30$ & $>30$ & $>30$ & & $>30$ & $>30$ & $>30$ & $>30$ & $>30$ & $>30$ & $>30$ & $>30$ & 111-120 \\
\hline 171-180 & $20-30$ & \begin{tabular}{|l|}
$20-30$ \\
\end{tabular} & 20-30 & $20-30$ & $>30$ & $>30$ & $>30$ & $>30$ & & $>30$ & $>30$ & $>30$ & $>30$ & $>30$ & $>30$ & $>30$ & $>30$ & \begin{tabular}{|l|}
$171-180$ \\
\end{tabular} \\
\hline 161-170 & $10-20$ & $20-30$ & $20-30$ & $20-30$ & $>30$ & $>30$ & $>30$ & $>30$ & & $>30$ & $>30$ & $>30$ & $>30$ & $>30$ & $>30$ & $>30$ & $>30$ & 161-170 \\
\hline $151-160$ & $10-20$ & $10-20$ & $20-30$ & $20-30$ & $20-30$ & $>30$ & $>30$ & $>30$ & & $>30$ & $>30$ & $>30$ & $>30$ & $>30$ & $>30$ & $>30$ & $>30$ & $151-160$ \\
\hline 141-150 & $10-20$ & $10-20$ & $10-20$ & $20-30$ & $20-30$ & $20-30$ & $>30$ & $>30$ & 55 a & $20-30$ & $>30$ & $>30$ & $>30$ & $>30$ & $>30$ & $>30$ & $>30$ & $141-150$ \\
\hline $131-120$ & $10-20$ & $10-20$ & $10-20$ & $20-30$ & $20-30$ & $20-30$ & $20-30$ & $>30$ & & $20-30$ & $20-30$ & $>30$ & $>30$ & $>30$ & $>30$ & $>30$ & $>30$ & $131-120$ \\
\hline $121-130$ & $10-20$ & $10-20$ & $10-20$ & $10-20$ & $20-30$ & $20-30$ & $20-30$ & $>30$ & & $20-30$ & $20-30$ & 20-30 & $>30$ & $>30$ & $>30$ & $>30$ & $>30$ & $121-130$ \\
\hline $111-120$ & $10-20$ & $10-20$ & $10-20$ & $10-20$ & $10-20$ & $20-30$ & $20-30$ & \begin{tabular}{|l|}
$20-30$ \\
\end{tabular} & & 20-30 & $20-30$ & $\mid 20-30$ & $20-30$ & $>30$ & $>30$ & $>30$ & $>30$ & $111-120$ \\
\hline 171-180 & 10-20 & \begin{tabular}{|l|}
$10-20$ \\
\end{tabular} & \begin{tabular}{|l|}
$10-20$ \\
\end{tabular} & 10-20 & $10-20$ & $20-30$ & $20-30$ & \begin{tabular}{|l|}
$20-30$ \\
\end{tabular} & & $20-30$ & $20-30$ & \begin{tabular}{|l|}
$20-30$ \\
\end{tabular} & $20-30$ & $>30$ & $>30$ & $>30$ & $>30$ & \begin{tabular}{|l|l}
$171-180$ \\
\end{tabular} \\
\hline 161-170 & \begin{tabular}{|l|}
$10-20$ \\
\end{tabular} & \begin{tabular}{|l|}
$10-20$ \\
\end{tabular} & \begin{tabular}{|c|}
$10-20$ \\
\end{tabular} & $10-20$ & \begin{tabular}{ll|}
$10-20$ \\
\end{tabular} & $10-20$ & $20-30$ & \begin{tabular}{|l|}
$20-30$ \\
\end{tabular} & & \begin{tabular}{|l|}
$10-20$ \\
\end{tabular} & 20-30 & \begin{tabular}{|l|}
$20-30$ \\
\end{tabular} & $20-30$ & $>30$ & $>30$ & $>30$ & $>30$ & |161-170 \\
\hline $151-160$ & 5-10 & 10-20 & $10-20$ & $10-20$ & $10-20$ & $10-20$ & $10-20$ & \begin{tabular}{|l|}
$20-30$ \\
\end{tabular} & & $10-20$ & $10-20$ & \begin{tabular}{|l|}
$20-30$ \\
\end{tabular} & $20-30$ & $20-30$ & $>30$ & $>30$ & $>30$ & $151-160$ \\
\hline $141-150$ & 5-10 & 5-10 & \begin{tabular}{|l|}
$10-20$ \\
\end{tabular} & $10-20$ & $10-20$ & $10-20$ & $10-20$ & \begin{tabular}{|l|}
$20-30$ \\
\end{tabular} & $45 \mathrm{a}$ & $10-20$ & $10-20$ & $10-20$ & $20-30$ & $20-30$ & $20-30$ & $>30$ & $>30$ & 141-150 \\
\hline $131-120$ & 5-10 & $5-10$ & 5-10 & $10-20$ & $10-20$ & $10-20$ & $10-20$ & \begin{tabular}{|l|}
$10-20$ \\
\end{tabular} & & $10-20$ & $10-20$ & 10-20 & $10-20$ & $20-30$ & $20-30$ & $20-30$ & $>30$ & \begin{tabular}{|l}
$131-120$ \\
\end{tabular} \\
\hline $121-130$ & $5-10$ & $5-10$ & $5-10$ & $5-10$ & $10-20$ & $10-20$ & $10-20$ & 10-20 & & $10-20$ & $10-20$ & $\mid 10-20$ & $10-20$ & $20-30$ & $20-30$ & $20-30$ & $>30$ & $121-130$ \\
\hline $111-120$ & $5-10$ & $5-10$ & $5-10$ & $5-10$ & $10-20$ & $10-20$ & $10-20$ & $10-20$ & & $10-20$ & $10-20$ & $|10-20|$ & $10-20$ & $10-20$ & $20-30$ & $20-30$ & 20-30 & $111-120$ \\
\hline 171-180 & $5-10$ & 5-10 & 5-10 & $5-10$ & 5-10 & $10-20$ & $10-20$ & \begin{tabular}{|l|}
$10-20$ \\
\end{tabular} & & $5-10$ & $10-20$ & \begin{tabular}{|l|}
$10-20$ \\
\end{tabular} & 10-20 & $10-20$ & $10-20$ & $20-30$ & $20-30$ & 171-180 \\
\hline 161-170 & $<5$ & $5-10$ & 5-10 & 5-10 & 5-10 & $5-10$ & $10-20$ & \begin{tabular}{|c|}
$10-20$ \\
\end{tabular} & & \begin{tabular}{|l|}
$5-10$ \\
\end{tabular} & $5-10$ & \begin{tabular}{|c|}
$10-20$ \\
\end{tabular} & $10-20$ & $10-20$ & 10-20 & $10-20$ & $20-30$ & 161-170 \\
\hline $151-160$ & $<5$ & $<5$ & $5-10$ & $5-10$ & $5-10$ & $5-10$ & $5-10$ & \begin{tabular}{|l|}
$10-20$ \\
\end{tabular} & & $5-10$ & $5-10$ & |10-20 & 10-20 & $10-20$ & $10-20$ & $10-20$ & $10-20$ & $151-160$ \\
\hline $141-150$ & $<5$ & $<5$ & $<5$ & $5-10$ & $5-10$ & $5-10$ & $5-10$ & $5-10$ & 35 a 44 & $5-10$ & $5-10$ & $5-10$ & $10-20$ & $10-20$ & $10-20$ & $10-20$ & $10-20$ & $141-150$ \\
\hline $131-120$ & $<5$ & $<5$ & $<5$ & $5-10$ & $5-10$ & $5-10$ & $5-10$ & $5-10$ & & $5-10$ & $5-10$ & $5-10$ & $5-10$ & $10-20$ & $10-20$ & $10-20$ & $10-20$ & $131-120$ \\
\hline $121-130$ & $<5$ & $<5$ & $<5$ & $<5$ & $5-10$ & $5-10$ & $5-10$ & $5-10$ & & $5-10$ & $5-10$ & $5-10$ & 5-10 & $10-20$ & $10-20$ & $10-20$ & $10-20$ & $121-130$ \\
\hline $111-120$ & $<5$ & $<5$ & $<5$ & $<5$ & $<5$ & $5-10$ & $5-10$ & $5-10$ & & $5-10$ & $5-10$ & $5-10$ & $5-10$ & $5-10$ & $10-20$ & $10-20$ & $10-20$ & $111-120$ \\
\hline & $\begin{array}{c}15 \mathrm{a} \\
20\end{array}$ & \begin{tabular}{|c|}
$20 \mathrm{a}$ \\
25
\end{tabular} & $\begin{array}{c}25 \mathrm{a} \\
30\end{array}$ & $\begin{array}{c}30 \mathrm{a} \\
40\end{array}$ & $\begin{array}{c}15 \mathrm{a} \\
20\end{array}$ & $\begin{array}{c}20 \mathrm{a} \\
25\end{array}$ & $\begin{array}{c}25 \mathrm{a} \\
30\end{array}$ & $\begin{array}{c}30 \mathrm{a} \\
40\end{array}$ & & $\begin{array}{c}15 \mathrm{a} \\
20\end{array}$ & $\begin{array}{c}20 \mathrm{a} \\
25\end{array}$ & $\begin{array}{c}25 \mathrm{a} \\
30\end{array}$ & $\begin{array}{c}30 \mathrm{a} \\
40\end{array}$ & $\begin{array}{c}15 \mathrm{a} \\
20\end{array}$ & $\begin{array}{c}20 \mathrm{a} \\
25\end{array}$ & $\begin{array}{c}25 \mathrm{a} \\
30\end{array}$ & $\begin{array}{c}30 \mathrm{a} \\
40\end{array}$ & \\
\hline & & & & & & & & & & & & & & & & & & \\
\hline
\end{tabular}


Las intervenciones para reducir el riesgo cardiovascular La mayoría de la evidencia sobre los riesgos y beneficios potenciales de las intervenciones para reducir el riesgo CV proviene de países de altos ingresos. Sin embargo, limitados datos epidemiológicos de tipo observacional como los provenientes del estudio Interheart ${ }^{17}$ avalan que los factores de riesgo tienen valor pronóstico similar en un amplio rango de condiciones geográficas y socioeconómicas; lo que hace suponer que la evidencia relacionada al descenso de los factores de riesgo también pueda extrapolarse.

Durante 2007 la Organización Mundial de la Salud (OMS) publicó un documento de toma de posición enfocado hacia en la evaluación y el manejo de individuos con aterosclerosis asintomática sobre la base de la estimación de su riesgo CV absoluto, definido como la probabilidad de que sufra un infarto agudo de miocardio (IAM) o un accidente cerebrovascular (ACV) durante cierto período, por ejemplo diez años.

La OMS también propone complementar este tipo de enfoque basado en el riesgo $\mathrm{CV}$ individual con estrategias de alcance poblacional, ya que si bien los eventos ocurren con mayor probabilidad en las personas de alto riesgo, no existe un nivel de riesgo que pueda considerarse seguro, ya que los eventos CV seguirán ocurriendo en las personas de "bajo riesgo", que justamente son la mayoría de la población (ver tabla 4). Este tipo de abordaje ha probado ser efectivo en los países de mayor desarrollo, habiendo demostrado moderar entre 1960 y 1990 sus epidemias de enfermedad CV -un ejemplo clásico es el de la experiencia de North Karelia, Finlandia.

Aclara además que para los decisores de salud es difícil establecer el umbral de riesgo CV al que llamarán "alto riesgo" y definir qué pacientes serán candidatos a intervenciones intensivas. Por convención y de acuerdo a la disponibilidad de recursos este umbral suele situarse cercano a un $30 \%$ de riesgo de un resultado combinado de eventos coronarios fatales y no fatales. Ver tabla 4. Al igual que otros documentos previos, la guía de la OMS incluye recomendaciones generales para la promoción de un estilo de vida sano que resumimos a continuación:

- Tabaquismo: aconsejar a los no fumadores a no comenzar a fumar y a no consumir tabaco en forma alguna, ofrecer ayuda a los fumadores para dejar de fumar (recomendaciones de tipo A) y recomendar el uso bupropion o nortriptilina y/o sustitutos de nicotina a los fumadores de mediano y alto riesgo que no hayan logrado dejar de fumar por sus propios medios (tipo B). - Alimentación: aconsejar a todos los individuos a reducir la ingesta de grasas saturadas, a eliminar la de ácidos grasos trans, a que la ingesta total de grasas no represente más de $30 \%$ de las calorías totales, a reducir la ingesta de sal (cloruro de sodio) a un máximo de $5 \mathrm{~g}$ por día y a comer un mínimo de
$400 \mathrm{~g}$ día de distintas variedades de vegetales y cereales de grano entero (A).

- Actividad física: aconsejar a todos los individuos a realizar un mínimo de 30 minutos diarios de actividad física moderada (ej. caminata rápida) en forma recreativa, o bien como tareas laborales $(A)$.

- Prevención de la obesidad: aconsejar a todos los individuos obesos o con sobrepeso a perder peso a través de una dieta reducida en energía y un aumento de la actividad física $(A)$.

- Recomendar: la disminución de la ingesta de alcohol a todas las personas que tomen tres o más unidades diarias (B).

- Disglucemia y síndrome metabólico: indicación de metformina en las personas que continúan con una glucemia superior a $110 \mathrm{mg} / \mathrm{dL}$ luego de intervenciones no farmacológicas $(A)$.

Se expide también en contra de la utilización de estrategias de eficacia no probada o que cuentan con evidencia de que no son de utilidad para la prevención de la enfermedad CV como la terapia de reemplazo hormonal y/o la suplementación con vitaminas $B, C$ y/o ácido fólico. Además y en consonancia con los consensos que mencionamos previamente, recomienda una serie de intervenciones individualizadas de acuerdo al perfil de riesgo CV de sujeto en cuestión y que hemos resumido en la tabla 5, comparándolas con las de los consensos anteriores, que son algo más agresivas, especialmente en la instauración de fármacos anti-hipertensivos e hipolipemiantes.

\section{Conclusiones}

Teniendo en cuenta la finitud de los recursos económicos, especialmente los relacionados al área de la salud, la OMS propone estrategias más conservadoras para los grupos de bajo riesgo $\mathrm{CV}$ global que las que habíamos resumido en nuestro primer artículo durante 2003; lo que se traduce en que, a similares niveles de riesgo CV global, proponga metas más modestas de tensión arterial y de lípidos, y por lo tanto, menor agresividad en los tratamientos farmacológicos, básicamente anti-hipertensivos e hipolipemiantes. Si bien y como nos ocurre muchas veces durante el ejercicio de nuestra profesión, esta toma de posición puede conducir a la recomendación estrategias y metas diferentes según contextos sanitarios de países de bajos o mayores recursos; celebramos que las recomendaciones de diagnostico y tratamiento se adapten a las realidades locales de cada país. Para ello, no sólo tienen que poder ser realizadas y estar disponibles, sino que deben implicar un buen uso de los recursos del sistema sanitario. Por ejemplo, tratar con estatinas a personas de bajo riesgo puede ser costo-efectivo en países de altos ingresos pero es claramente menos costo-efectivo en países menos afluentes. Incorporar explícitamente nociones de costo-efectividad con las guías de practica clínica, es un avance en esa dirección.

Tabla 4: distribución de la población por edad y sexo según su riesgo cardiovascular global a diez años en la región B de las Américas.

\begin{tabular}{|c|c|c|c|c|c|c|c|c|c|}
\hline \multicolumn{5}{|c|}{ Hombres } & \multicolumn{5}{|c|}{ Mujeres } \\
\hline & \multicolumn{4}{|c|}{ Edad en años } & \multirow{2}{*}{$\begin{array}{l}\text { Riesgo cardiovascular } \\
\text { global a diez años }\end{array}$} & \multicolumn{4}{|c|}{ Edad en años } \\
\hline & Menos de 50 & 50 a 59 & 60 a 69 & Más de 70 & & Menos de 50 & 50 a 59 & 60 a 69 & Más de 70 \\
\hline Menos de $10 \%$ & $96 \%$ & $86 \%$ & $64 \%$ & $43 \%$ & Menos de $\mathbf{1 0} \%$ & $98 \%$ & $83 \%$ & $64 \%$ & $42 \%$ \\
\hline 10 a $20 \%$ & $3 \%$ & $8 \%$ & $13 \%$ & $29 \%$ & 10 a $20 \%$ & $2 \%$ & $12 \%$ & $7 \%$ & $20 \%$ \\
\hline 20 a $30 \%$ & \multirow{3}{*}{ Menos de $0,3 \%$} & $4 \%$ & $12 \%$ & $15 \%$ & 20 a $30 \%$ & \multirow{3}{*}{ Menos de $0,4 \%$} & $4 \%$ & $21 \%$ & $13 \%$ \\
\hline 30 a $40 \%$ & & $1 \%$ & $8 \%$ & $9 \%$ & 30 a $40 \%$ & & $1 \%$ & $6 \%$ & $20 \%$ \\
\hline Más de $\mathbf{4 0} \%$ & & $0,5 \%$ & & $\%$ & Más de $\mathbf{4 0} \%$ & & $0,4 \%$ & $3 \%$ & $5 \%$ \\
\hline
\end{tabular}

Región B de las Américas: incluye Antigua y Barbuda, Argentina, Bahamas, Barbados, Bélice, Brasil, Chile, Colombia, Costa Rica, Dominica, República Dominicana, El Salvador, Grenada, Guyana, Honduras, Jamaica, México, Panamá, Paraguay, San Kitts y Nevis, Santa Lucia, San Vincente y Grenadinas, Surinam, Trinidad y Tobago, Uruguay, Venezuela.

Fuente: Prevention of cardiovascular disease: guidelines for assessment and management of total cardiovascular risk. Guidelines. I. World Health Organization. ISBN 9789241547178 (NLM classification: WG 120). @ World Health Organization 2007. 
Tabla 5: guía orientadora para la toma de decisiones sobre intervenciones farmacológicas según el riesgo absoluto de desarrollo de enfermedad cardiovascular de cada paciente.

\begin{tabular}{|c|c|c|}
\hline \multirow{2}{*}{$\begin{array}{l}\text { Subpoblación por co-morbilidades o riesgo } \\
\text { absoluto de desarrollar enfermedad coronaria }\end{array}$} & \multicolumn{2}{|c|}{ Intervenciones farmacológicas mas allá de las destinadas a promover mejoras en el estilo de vida } \\
\hline & Abordaje agresivo $^{a}$ & Abordaje conservador \\
\hline Bajo (menor a $10 \%$ a diez años) & $\begin{array}{l}\text { Antihipertensivos si la TA supera } 140 / 90 \mathrm{mmHg} \text {. } \\
\text { Estatinas si el colesterol LDL supera } 160 \mathrm{mg} / \mathrm{dL}\end{array}$ & $\begin{array}{l}\text { Antihipertensivos si la TA supera } 160 / 110 \mathrm{mmHg} \text { (B). } \\
\text { Estatinas si el colesterol total supera } 320 \mathrm{mg} / \mathrm{dL} \text { (B). }\end{array}$ \\
\hline Intermedio (10 a $20 \%$ a diez años) & $\begin{array}{l}\text { Antihipertensivos si la TA supera } 140 / 90 \mathrm{mmHg} \text {. } \\
\text { Estatinas si el colesterol LDL supera } 130 \mathrm{mg} / \mathrm{dL} \text {. } \\
\text { Aspirina }\end{array}$ & $\begin{array}{l}\text { Antihipertensivos si la TA supera } 160 / 110 \mathrm{mmHg}(\mathrm{B}) \text {. } \\
\text { Estatinas si el colesterol total supera } 320 \mathrm{mg} / \mathrm{dL} \text { (B). }\end{array}$ \\
\hline Alto (20 a $30 \%$ a diez años) & Antihipertensivos si la TA supera $130 / 80 \mathrm{mmHg}$. & Antihipertensivos si la TA supera 140/90 mmHg (A). \\
\hline \multirow{2}{*}{$\begin{array}{l}\text { Síndrome metabólico; diabetes de menos de diez años } \\
\text { de evolución, sin daño de órgano blanco y con buen } \\
\text { control; un factor de riesgo muy severo; hipertensión } \\
\text { arterial con daño de órgano blanco. }\end{array}$} & $\begin{array}{c}\text { Estatinas si el colesterol LDL supera } 100 \mathrm{mg} / \mathrm{dL} 0 \text { el } \\
\text { equivalente a } 40 \mathrm{mg} \text { de sinvastatina a todos. } \\
\text { Aspirina }\end{array}$ & $\begin{array}{c}\text { Estatinas si el colesterol total supera } 200 \mathrm{mg} / \mathrm{dL} \text { y/0 } \\
\text { el LDL } 120 \mathrm{mg} / \mathrm{dL}(\mathrm{A}) \\
\text { Opcionalmente aspirina }\end{array}$ \\
\hline & \multicolumn{2}{|c|}{ Metformina si hay disglucemia } \\
\hline \multirow[t]{3}{*}{$\begin{array}{l}\text { Enfermedad cardiovascular establecida; diabetes de más } \\
\text { de diez años de evolución o con daño de órgano } \\
\text { blanco o con mal control; dislipemias familiares o } \\
\text { genéticas; riesgo extremadamente alto por múltiples } \\
\text { factores de riesgo; síndrome coronario agudo. }\end{array}$} & $\begin{array}{l}\text { Estatinas si el colesterol supera los } 70 \mathrm{mg} / \mathrm{dL} 0 \text { un mínimo } \\
\text { de } 40 \mathrm{mg} \text { de sinvastatina } 0 \text { su equivalente a todos. } \\
\text { IECA en pacientes vasculares y diabéticos. }\end{array}$ & $\begin{array}{l}\text { Estatinas si el colesterol total supera } 200 \mathrm{mg} / \mathrm{dL} \text { y/0 } \\
\text { el LDL } 120 \mathrm{mg} / \mathrm{dL} \text { (A). }\end{array}$ \\
\hline & \multicolumn{2}{|c|}{ Aspirina $(A)$} \\
\hline & \multicolumn{2}{|c|}{ Metformina si hay disglucemia } \\
\hline
\end{tabular}

TA: tension arterial. IECA: inhibidores de la enzima convertidora de angiotensina. OMS: Organización Mundial de la Salud. Disglucemia: alteraciones del metabolismo de la glucosa en pacientes que no cumplen criterios de diabetes y que incluyen a la glucemia alta en ayunas (mayor a $110 \mathrm{mg} / \mathrm{dL}$ ) y a la alteración de la tolerancia oral a la glucosa.

a El abordaje "agresivo" fue esquematizado sobre la base de las recomendaciones de los siguientes consensos: New Zealand Guidelines Group, National Heart Foundation of New Zealand and Stroke Foundation of New Zealand. Best Practice Evidence-based Guideline. The assessment and management of cardiovascular risk. 2003, New Zealand Guidelines Group; National Collaborating Centre for Chronic Conditions. Hypertension: management of hypertension in adults in primary care: partial update. London: Royal College of Physicians,2006. National Cholesterol Education Program (NCEP) Expert Panel on Detection, Evaluation, and Treatment of High Blood Cholesterol in Adults (Adult Treatment Panel III). Third Report of the National Cholesterol Education Program (NCEP) Expert Panel on Detection, Evaluation, and Treatment of High Blood Cholesterol in Adults (Adult Treatment Panel III) final report. Circulation. 2002;106:3143-3421. Grundy S y col.; for the Coordinating Committee of the National Cholesterol Education Program. NCEP report. Implications of Recent Clinical Trials for the National Cholesterol Education Program Adult Treatment Panel III Guidelines. Circulation. 2004; 110:227-239. Cooper A y col. (2007) Clinical Guidelines and Evidence Review for Lipid Modification: cardiovascular risk assessment and the primary and secondary prevention of cardiovascular disease London: National Collaborating Centre for Primary Care and Royal College of General Practitioners. National Collaborating Centre for Chronic Conditions. Type 2 diabetes: national clinical guideline for management in primary and secondary care (update). London: Royal College of Physicians, 2008.

b El abordaje "conservador" fue esquematizado sobre la base de las recomendaciones de la Organización Mundial de la Salud explicitadas en su documento: Prevention of cardiovascular disease: guidelines for assessment and management of total cardiovascular risk. Guidelines. I. World Health Organization. ISBN 9789241547178 (NLM classifi cation: WG 120). ( ) World Health Organization 2007.

Recibido el 23/05/08 y aceptado el 11/06/08

\section{Referencias}

1. Mendis S et al. for the Pathobiological Determinants of Atherosclerosis in Youth (PBDAY) Research group. Atherosclerosis in children and young adults: An overview of the World Health Organization (WHO) and Intemational Society and Federation of Cardiology Study on Pathobiological Determinants of Atherosclerosis in Youth study (1985-1995). Prevention and Control, 2005;1:3-15.

2. Schargrodsky H. CARMELA: Assessment of Cardiovascular Risk in Seven Latin American Cities American Journal of Medicine (2008) 121, 58-65.

3. Kopitowski K, Terrasa S. Riesgo vascular global (primera parte).Evidencia en Atención Primaria. Vol 6 No5.Sept-Octubre de 2003.Pag 152. Nov.de 2003. Disponible en intemet: http://www.foroaps.org/hitalba-pagina-articulo.php?cod_producto=1241\&vol=6\&nr_bi=5\&ano=2003 (útimo acceso 13/05/08).

4. Terrasa S, Kopitowski K. Evaluación del riesgo global (segunda parte). Evidencia Actualización en la Práctica Ambulatoria - Vol. 7 Número 2: Marzo-Abril 2004. Disponible en Intemet: http://mww.foroaps.org/hitallba-paginaarticulo.php?cod_producto=1344\&vol=7\&nr_bi=2\&ano=2004 (último acceso 13/05/08). http:/www.foroaps.orgffiles/riesgo\%20global\%202.pdf

5. Grundy S, Pasternak R, Greenland P, et al. Assessment of cardiovascular Risk by Use of Multiple-Risk factor Assessment Equations. A Statement for Healthcare Professionals From the American Heart Association and the American College of Cardiology. J Am Coll Cardiol 1999; 34:1348-59.

6. Assmann G, Cullen P, Schulte H.Simple scoring scheme for calculating the risk of acute coronary events based on the 10-year follow-up of the prospective cardiovascular Munster (PROCAM) study. Circulation 2002 Feb 19;105(7):900. 7. Conroy R, Pyörälä K, Fitzgerald A, Sans S, Menotti A, De Backer G, et. al. on behalf of the SCORE project group. Estimation of ten years risk of fatal cardiovascular disease in Europe: the SCORE project. European Heart Journal (2003) 24, 987-1003.

8. National Cholesterol Education Program (NCEP) Expert Panel on Detection, Evaluation, and Treatment of High Blood Cholesterol in

Adults (Adult Treatment Panel III). Third Report of the National Cho lesterol Education Program (NCEP) Expert Panel on Detection, Evaluations

Adults (Adult Treatment Panel III). Third Report of the National Cho lesterol Education Program (NCEP) Expert Panel on Detection, Evaluiti 9. Grundy S y col.; for the Coordinating Committee of the National Cholesterol Education Program. NCEP report. Implications of Recent Clinical Trials for the National Cholesterol Education Program Adult Treatment Panel III Guidelines. Circulation. 2004;110:227-239. Disponible en URL: http://www.nhlbi.nih.gov/guidelines/cholesterol/atp3upd04.pdf

(ultimo acceso 23/05/08

10. New Zealand Guidelines Group, National Heart Foundation of New Zealand and Stroke Foundation of New Zealand. Best Practice Evidence-based

Guideline. The assessment and management of cardiovascular risk. 2003, New Zealand Guidelines Group. Disponible en intemet: www.nzgg.org.nz (ultimo acceso 13/05/08).

11. 2003 European Society of Hypertension-European Society of Cardiology guidelines for the management of arterial hypertension. Guidelines Committee. Journal of Hypertension 2003, 21:1011-1053

12. Mendis S; Lindholm L, Mancia G y col. World Health Organization (WHO) and Intemational Society of Hypertension (ISH) risk prediction charts: assessment of cardiovascular risk for prevention and control of cardiovascular disease in low and middle-income countries. Journal of Hypertension. 25(8):1578-1582, August 2007.

13. National Collaborating Centre for Chronic Conditions. Hypertension: management of hypertension in adults in primary care: partial update. London: Royal College of Physicians, 2006. Disponible en Intemet: http://www.nice.org.uk/guidance/CG34 (útimo acceso 13/05/08).

14. Cooper A, Nherera L, Calvert N, y col. (2007) Clinical Guidelines and Evidence Review for Lipid Modification:cardiovascular risk assessment and the primary and secondary prevention of cardiovascular disease London: National Collaborating Centre for Primary Care and Royal College of General Practitioners. Disponible en URL: http://www.nice.org.uk/nicemedia/pdf/CG67fullguideline.pdf (útimo acceso 11/06/08).

15. National Collaborating Centre for Chronic Conditions. Type 2 diabetes: national clinical guideline for management in primary and secondary care (update). London: Royal College of Physicians, 2008. Disponible en URL: http://www.nice.org.uk/guidance/index.jsp?action=download\&o=40803 (último acceso 11/06/08)

16. World Health Organization. Prevención de enfermedades cardiovasculares. Guía de Bolsillo para la Estimación y el Manejo del Riesgo Cardiovascular: Americas. Ginebra,2007 ISBN-13 9789243547282 ISBN-10 924354728 En prensa: $h$ ttp://www. who.intbookorders/espagnoldetart3.jsp?sesslan=3\&codlan=3\&codcol=15\&codcch=4713\#

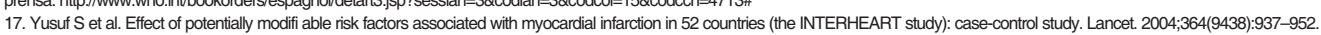

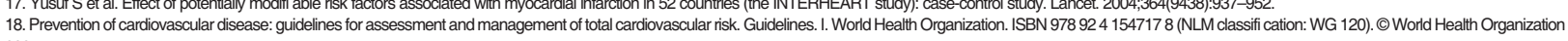
2007. 